\title{
ChR2 coming of age
}

\author{
10 years ago, channelrhodopsin- 2 was expressed in neurons and shown to control their activity. In this issue, we \\ consider how the field has developed since these early optogenetic experiments.
}

T his month, Nature Neuroscience celebrates the tenth anniversary of the publication of a study demonstrating that channelrhodopsin-2 (ChR2) could be used to control the electrical activity of neurons with millisecond precision. For the occasion, we invited members of the community to discuss the state of the field and comment on how optogenetics has affected their work (Q\&A on page 1202). We also hear from the first and senior authors of the 2005 paper. On page 1200, Ed Boyden provides an Overview that considers the future of optogenetics in neuroscience and, in a Historical Commentary on page 1213, Karl Deisseroth reflects on the past decade of optogenetic research. Online, the special issue is complemented by an editorially curated Web Collection featuring articles from the Nature journals reporting advances in the optogenetics toolkit and describing some of the discoveries that they have yielded.

ChR2 is now a ubiquitous tool in neuroscience research, but its addition to the neuroscience arsenal was only made possible through the work of scientists in the orthogonal field of photobiology. ChR2 was first isolated and characterized by Georg Nagel, Ernst Bamberg and Peter Hegemann, who were searching for the proteins responsible for photocurrents in the unicellular green alga Chlamydomonas reinhardtii. Building on this discovery and repurposing ChR2 from a sensor to an actuator, Ed Boyden, Feng Zhang and Karl Deisseroth, in collaboration with Nagel and Bamberg, showed that the light-gated channel could be used to control neural activity with high temporal precision. The fact that this microbial protein, a stranger in the animal kingdom, could readily be expressed in neurons, reach the plasma membrane and function normally was remarkable. Given what we now know, the 2005 paper marked a pivotal step in the development of optogenetics.

Putting ChR2 in neurons was a brilliant idea, but this was not the first attempt at controlling neurons with light. Earlier proof-of-concept studies from the Miesenböck and the Trauner and Kramer labs, to name the most visible efforts, also showed that light could be harnessed to control neuronal activity. These earlier optogenetics approaches almost certainly fueled the inspiration to pursue experiments with ChR2, which had perhaps originally been spurred by Francis Crick's visionary proposal for interrogating brain function (doi:10.1098/rstb.1999.0541 and see our Q\&A). ChR2's appeal over other genetically based approaches was twofold. It was a single-component solution to actuating the membrane potential and, in part as a consequence of this, its kinetics were orders of magnitude faster than those of its competitors, comparable with phenomena of keen interest such as synaptic events and action potentials.

In hindsight, it seems obvious that the 2005 paper reported a breakthrough. However, the paper's scope was relatively limited (neurons in a dish) and, even by the standards for high-profile publication back in 2005, the validation of the technique must have appeared perfunctory to readers. It should therefore not come as a surprise that, at the time the manuscript was evaluated, skepticism was high. After being rejected without review by both Nature and Science, the manuscript received mixed feedback at Nature Neuroscience. Although there was some tangible excitement about the potential of the technique, there were doubts regarding its utility. The reviewers requested greater characterization and evidence of generalizability to other experimental conditions, and one referee was quite negative, saying, "The most serious problem is that, while the method seems fancy and somehow promising, there is to my eyes no obvious question requiring its use." The recently introduced Technical Report format offered a path forward to publication, as new biological findings were not a requirement, and a revision was invited by the editors. The revised paper was accepted, but some referees still had doubts. In their comments to the editors, one stated, "[...] I think the technique is novel and has exciting and important prospects for future use. However, [...] my enthusiasm for the paper is only moderate because until (or unless) it can be shown to be useful in a slice preparation, it is pretty useless." Another indicated, "Although the immediate application of the method is still unclear, I feel now that in view of the elegance of the technique the manuscript can be published as a 'Technical note." Although this skepticism reflects the fact that revolutionary findings can be hard to appreciate when they first emerge, it is also fair to say that some uncertainty about their potential was justified, especially given that previous approaches had yet to see broad uptake. Before ChR2 could be widely adopted, more substantial evidence of its utility was required.

Soon after the publication of the 2005 paper, several studies demonstrating how ChR2 could be used for behavioral control appeared rapidly, driven in part by competitive fire, but also inspired by the ease of use of ChR2, a key feature that was critical for its widespread adoption. It wasn't long before we saw huge conceptual leaps from the original experiments in cultured neurons and the conversion of many (but likely not all) skeptics. At the same time, the toolkit was expanding; most importantly, to include 'silencers' that complemented ChR2's stimulatory activity. Dissemination was bolstered by broad sharing of reagents, thereby creating a vibrant community of enthusiasts boldly exploring new kinds of experiments. As with all techniques, optogenetics has its limitations, but there is no doubt that it has propelled the field forward, particularly by being a strong catalyst for circuit-level analyses. The field of optogenetics is still growing, but, a decade in, has reached a certain maturity. In preparing this issue, we have enjoyed contemplating both how the field has progressed in the 10 years since ChR2's introduction to neurons and how it will continue to evolve from here. It's exciting to imagine what we might have to say about optogenetics 10 years from now, and we hope that you look forward to its future as much as we do. 